movements of either the left eye or the right eye (see figure; Zhou-King dashed rectangle).

A curious fact is that the presumed left and right eye information conveyed by the EBNs, the velocity component of the eye movement, is lost on their target cells in the abducens nuclei. The same argument can be made for the neurons in the nucleus prepositus hypoglossi (NPH) ${ }^{1}$, located just caudal to the abducens nucleus. These NPH neurons are thought to provide abducens neurons with an eye position signal obtained by integration of the velocity signal from the EBNs (ref. 7). Zhou and King describe the activity in most lateral rectus motoneurons as 'binocular' because most of the neurons are not uniquely related to the movement of either eye alone. Although described in different terms, this observation complements an earlier report ${ }^{2}$ on the activity of AI neurons and by inference, the lateral rectus motoneurons. When one transforms the conjugate and vergence indices used by Gamlin et al. ${ }^{2}$ to left/right eye measurements, the same pattern of signals described by Zhou and King for lateral rectus motoneurons is seen for identified AI neurons. Stated sim. ply, the left eye and right eye signals are commingled so that the net effect is that both $\mathrm{AI}$ neurons and lateral rectus motoneurons receive the same signal, which is essentially a conjugate signal with a bias toward the ipsilateral eye. Why separate left eye/right eye signals should be synthesized and then discarded at the level of the abducens nucleus is difficult to understand. Neither symmetrical vergence nor changes in vergence accomplished by unequal saccades can be controlled directly by right eye/left eye EBNs or NPH inputs to the abducens nucleus because this information is lost at the target neurons. Zhou and King suggest that the left eye/right eye signals from EBNs and NPH neurons may be related to an evolutionary heritage of eyes that move independently ${ }^{1}$.

The bottom line is that whether one chooses the Hering or the Zhou-King scheme, the signals reaching the abducens and oculomotor nuclei are the same, and a vergence signal (near response signal) must still be provided to the motoneurons controlling activity of the contralateral medial rectus musclean observation consistent with Hering's Law. Nonetheless, Zhou and King have reported an unexpected result, one that will prompt a reexamination of our basic assumptions about oculomotor circuits.

\section{Acknowledgement}

I thank Claudio Busettini for his valuable

discussions and help with the figure.

1. Zhou, W. \& King, W.M. Premotor commands en code monocular eye movements. Nature 393 , 692-695 (1998).

2. Gamlin, P.D.R. Gnadt, J.W. \& Mays, L.E. Abducens internuclear neurons carry an inappropriate signal for ocular convergence. ). Neurophysiol. 62, 70-81 (1989).

3. Mays, L.E. Neural control of vergence eye move ments: convergence and divergence neurons in the midbrain. /. Neurophysiol. 51, 1091-1108 (1984).

4. Zhang, Y., Mays, L.E. \& Gamlin, P.D.R. Characteristics of near response cells projecting to the oculomotor nucleus. \%. Neurophysiol. 67, 944-960 (1992).

5. Zee, D.S., FitzGibbon, E.J. \& Optican, L.M. Saccadevergence interactions in humans. J. Neurophysiol. 68 1624-1641 (1992)

6. Maxwell, J.S. \& King, W.M. Dynamics and efficacy of saccade-facilitated vergence eye movements in monkeys. J. Neurophysiol. 68, 1248-1260 (1992).

7. Cannon, S.C. \& Robinson, D.A. Loss of the neural integrator of the oculomotor system from brainstem lesions in monkey. J. Neurophysiol 57, 1383-1409 (1987).

Department of Physiological Optics

University of Alabama at Birmingham

Birmingham, Alabama 35294, USA

email:lmays@uab.edu

\title{
Spirochetes come in from the cold
}

\section{Spirochetes alter their major surface proteins in response to the temperature change that accompanies their} passage from tick vector to mammalian host.

$\mathrm{R}_{\mathrm{e}}^{\mathrm{s}}$ ELAPSING FEVER IS an ancient human disease characterized by a rise and fall in fever that was recognized by Hippocrates ${ }^{1}$. The etiologic agents of relapsing fever are spirochete bacteria of the genus Borrelia (see figure), and their vectors are blood-sucking lice and ticks. Lyme disease, caused by Borrelia burgdorferi, is the most common arthropodborne disease in Europe and the United States. Although Robert Koch and other early microbiologists studied the life history of Borrelia species in their louse and tick vectors ${ }^{1}$, most research over the past half century has focused on spirochetes in their rodent and human hosts. An article by Schwan and Hinnebusch ${ }^{2}$ in a recent issue of Science reminds us that these pathogens have an equally important life cycle inside arthropods. These investigators show that Borrelia hermsii, which causes relapsing fever in humans, alters its major surface proteins as it moves from tick vector to mammalian host and that this alteration is triggered

\section{ALAN G. BARBOUR}

in part by the temperature difference between ticks and mammals.

Fast-feeding ticks of the genus Ornithodoros (see figure) are the vectors of $B$. hermsii, which is responsible for outbreaks of relapsing fever in western North America. While residing in its mammalian host, $B$. hermsii expresses several different surface membrane-anchored lipoproteins, the variable major proteins (VMP), which determine the serotype of the pathogen ${ }^{3}$. Host antibodies produced in response to the infection select against those spirochetes expressing the predominant VMP (ref. 4). The few remaining spirochetes that produce different VMPs are not affected by these antibodies. These minority populations proliferate in the blood causing fever until they are cleared by a new round of host antibodies. There may be up to ten rounds of spirochete proliferation and fever during a single infection and with each relapse a different Borrelia serotype predominates in the blood. This Borrelia characteristic, which enables the pathogen to evade the host immune response, is termed multiphasic antigenic variation and its genetic basis is at least in part understood ${ }^{5,6}$.

Schwan and Hinnebusch inoculated mice with either of two antigenically distinct serotypes (determined by VMP7 or VMP8 proteins) of B. hermsii. They then allowed ticks to feed upon the spirochetemic mice and to become infected. The investigators found to their surprise that the spirochetes in the ticks all expressed a new serotype antigen, VMP33, regardless of whether they originally came from serotype 7- or serotype 8 -infected mice. When these Borreliabearing ticks were used to infect a second group of mice, the spirochetes again expressed either VMP7 or VMP8, depending on which serotype the original mice received. Thus, the VMP serotype could be reset to that present 

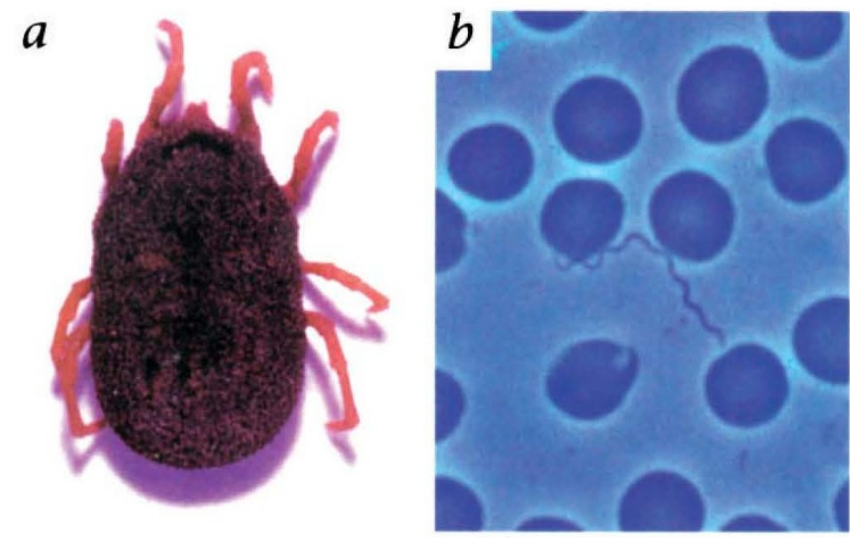

a, The soft tick

Ornithodoros turicata is a vector of spirochetes of the species Borrelia that cause relapsing fever (magnification $7 \times) . \boldsymbol{b}, \mathrm{A}$ spirochete in a blood smear from a patient with relapsing fever (Wright's stain; magnification $1600 x$ ).

before the spirochetes entered the tick.

Temperature is one environmental variable that seems to affect VMP expression. Schwan and Hinnebusch found that lowering the temperature for spirochete cultivation from $37^{\circ} \mathrm{C}$ to 23 ${ }^{\circ} \mathrm{C}$ (the typical temperature of ticks when not attached to mammals) increased the expression of VMP33 and decreased expression of mouse-associated VMP8. Although the authors do not characterize the function of either VMP33 or the mouse associated-VMPs, they suggest that these proteins may be important in transmission or colonization particularly because they are conserved among all Borrelia species.

The gene and promoter for the tick-associated VMP of $B$. hermsii are located on a different plasmid from the genes encoding the mouse-associated VMPs (ref. 7). How B. hermsii regulates expression of genes at two different loci is unknown. During serotype switching in the mouse, the expressed $v m p$ gene is replaced by a different $v m p$ gene on the same or different plasmids through DNA rearrangement ${ }^{5}$. In contrast, the gene for VMP33 does not undergo rearrangement: as expression from the tick-associated $v m p$ locus increases, expression of the mouse-associated $v m p$ gene appears to decrease (A.B., unpublished work).

The cyclical variation of VMP expression by $B$. hermsii is another example of a pathogen altering its phenotype as it moves from one type of host to another. Other infectious agents exhibiting such antigenic variation include those causing malaria, African trypanosomiasis and plague. $B$. burgdorferi, the etiologic agent of Lyme disease, expresses a completely different set of proteins in the tick and the mammal ${ }^{8,9}$. Outer surface protein (Osp) C, a B. burgdorferi protein that is homologous to VMP33 of $B$. hermsii ${ }^{7}$, is preferentially expressed in mammalian blood at $37{ }^{\circ} \mathrm{C}$ instead of at $23{ }^{\circ} \mathrm{C}$. Paradoxical though this seems, what VMP33 and OspC have in common (besides their sequence) is their association with tick salivary glands-the launching pads from which spirochetes invade their mammalian hosts. During the week-long blood meal of the $B$. burgdorferi tick vector, the spirochetes slowly travel from the intestine to the salivary glands, taking several days to reach their destination. Accordingly, expression of OspC is stimulated by the presence of blood in the tick intestine and the higher temperature close to the tick skin. On the other hand, $B$. hermsii spirochetes have to be present in the salivary glands of Ornithodoros ticks at the start of feeding because the tick blood meal only lasts an hour, too little time for the spirochetes to change surface antigens and move from the intestine to the salivary glands.

Several decades have passed since the last important studies of relapsing fever spirochetes in their tick vectors were published $^{10-12}$. Why has a line of research initiated by Koch and other pioneering scientists been neglected for so long? One reason might be because ticks are a difficult experimental model to work withlife cycles measured in years make rearing them in the laboratory tedious and experiments lengthy. Moreover, research funding and the training infrastructure for the discipline of medical entomology dwindled after World War II, a response to the temporary victories of antibiotics and pesticides. This decline now appears to be short-sighted, particularly given the new threats posed by emerging and reemerging vector-borne infections, such as Lyme disease and malaria.

The article by Schwan and Hinnebusch as well as other recent studies may represent a turning point for scientific interest in (and research funding of) tick-pathogen interactions ${ }^{13}$. A re- newed focus on the vectors of infection may yield new strategies to control and prevent arthropod-borne diseases. Contemporary investigators armed with the tools of molecular biology and genetics are successfully tackling problems that were first identified and described 50,100 , even 2,500 years ago.

1. Barbour, A.C. \& Hayes, S.F. Biology of Borrelia species. Microbiol Rev 50, 381-400 (1986).

2. Schwan, T.G. \& Hinnebusch, B.J. Bloodstream versus tick-associated variants of a relapsing fever bacterium. Science 280, 1938-1940 (1998).

3. Barbour, A.G. Linear DNA of Borrelia spp. and antigenic variation. Trends Microbiol. 1, 236-239 (1994).

4. Stoenner, H.G., Dodd, T. \& Larsen, C. Antigenic variation of Borrelia hermsii. /. Exp. Med. 156, 1297-1311 (1982).

5. Kitten, T. \& Barbour, A.G. Juxtaposition of expressed variable antigen genes with a conserved telomere in the bacterium Borrelia hermsii. Proc. Natl. Acad. Sci. USA 87, 6077-6081 (1990)

6. Restrepo, B.I. \& Barbour, A.G. Antigen diversity in the bacterium Borrelia hermsii through "somatic" mutations in rearranged $v m p$ genes. Cell 78 867-876 (1994)

7. Carter, C.J., Bergstrom, S., Norris, S.J. \& Barbour, A.G. A family of surface-exposed proteins of 20 kilodaltons in the genus Borrelia. Infect Immun 62, 2792-2799 (1994)

8. Schwan, T.G., Piesman, I., Golde, W.T., Dolan, M.C. \& Rosa, P.A. Induction of an outer surface protein on Borrelia burgdorferi during tick feeding. Proc. Natl. Acad. Sci. USA 92, 2909-2913 (1995).

9. de Silva, A.M., Telford, S.R., Brunet, L.R., Barthold, S.W. \& Fikrig, E. Borrelia burgdorferi OspA is an arthropod-specific transmission-blocking Lyme dis ease vaccine. J. Exp. Med. 183, 271-275 (1996).

10. Davis, G.E. The relapsing fevers: tick-spirochete specificity studies. Exp. Parasitol. 1, 406-410 (1952).

11. Burgdorfer, W. Analyse des Infektionsverlaufes be Ornithodorus moubata und der natürlichen Uebertragung von Spirochaeta duttoni. Acta Tropica 8, 196-262 (1951)

12. Aeschlimann, A. Développement embryonnaire d'Ornithodorus moubata (Murray) et transmission transovarienne de Borrelia duttoni. Acta Tropica 15, 15-64 (1958).

13. Schwan, T.G. Ticks and Borrelia: model systems for investigating pathogen-arthropod interactions. Infect. Agents Dis. 5, 167-181 (1996).

Departments of Microbiology \& Molecular Genetics and Medicine

University of California Irvine

Irvine, California 92612-4025, USA

email: abarbour@uci.edu

The complete genome sequence of a fellow spirochete, Treponema pallidum, the bacterium that causes syphilis, appears in the July 17 th issue of Science. The genome map compiled by Weinstock and Norris (University of Texas Health Sciences Center) and Fraser's group at The Institute for Genomic Research (Rockville, Maryland) identifies a number of novel Treponema genes, including 12 that encode surface membrane proteins which may be valuable as targets in vaccine development. Kristine Novak 